\title{
Imaging of Chemical Processes Via Electrical, Optical, and Ultrasonic Tomography
}

\author{
D. M. Scott, DuPont Company, Experimental Station E304/A212, Wilmington DE 19880-0304 USA
}

Over the past two decades a large number of tomographic methods have been developed for 3-D and 4-D imaging of industrial processes [1-2]. These on-line characterization methods are based on the measurement of electrical parameters (current, capacitance, or inductance) or the attenuation of various forms of energy. This data is used to reconstruct cross-sectional or full volume images of the distribution or concentration of the component phases in side the process vessel. Electrical and optical methods provide tomographic images in excess of several thousand frames per second [3-4] and can monitor dynamic processes. This technology provides unique data about the structure of multiphase materials over a wide range of length scales.

Optical tomography has been used in research engines to monitor distribution of hydrocarbons at the flame front during internal combustion cycles [3]. Optical fibers and collimation lenses are used to launch infrared light along 32 paths (at 4 separate angles) through the wall of a combustion chamber. Chemical species are distinguished by their infrared absorption, and by choosing laser diodes with the appropriate wavelength the distribution of various target species can be imaged. Cross-sectional images are reconstructed from the infrared absorption data using a model-based algorithm [5]. Up to 3,500 frames per second can be imaged. A sequence of images from this system is shown in Fig. 1.

Electrical Impedance Tomography (EIT) generates a cross-sectional image of conductivity variations in an opaque sample by measuring the impedance (or sometimes simply the resistance) between pairs of electrodes arranged around the perimeter of a material. Inhomogeneities are detected by measuring the impedance between every pair of electrodes and reconstructing a tomographic image of the conductivity [1]. Recently EIT was used to study water migration in ceramic pastes being extruded through $2 \mathrm{~mm}$ holes [6]. In this application spatial variations in the water content of the paste were measured using the sensor array in shown Fig. 2. It was found that a dry ring (see Fig. 3) forms around the wall of the extruder barrel during extrusion and affects the rheology of the paste. This is one of the smallest-scale applications of EIT realized to date.

Tomographic images can also be created from measurements of ultrasonic attenuation or phase shift, typically made in the frequency range 1-20 MHz. Applications range from medical diagnostics to nondestructive evaluation of materials to process monitoring. Industrial applications present a challenge due to the wide range of acoustic impedance encountered in the materials of interest and consequent issues with refraction and reflection of the ultrasonic beam. One system that has been demonstrated on industrial equipment uses a ring of 16 transducers to image the size and position of the air core inside a small (50 mm diameter) hydrocyclone [7]. The air core image can be seen in Fig. 4. This prototype has a frame rate of $6 \mathrm{~s}^{-1}$ and a resolution of a few millimeters.

The spatial resolution of these techniques is limited by the number and spacing of sensors, the nonlinearity inherent in "soft" sensing fields, and other effects. Iterative reconstruction methods (including model-based approaches) have been used to improve the image resolution or to extract useful information in spite of these limitations. These tomographic imaging and information extraction techniques are broadly applicable in other applications. 
References

[1] R. A. Williams and M. S. Beck, Process Tomography, Butterworth-Heinemann, 1995.

[2] D. M. Scott and H. McCann, Process Imaging for Automatic Control, Marcel Dekker 2005.

[3] F. P. Hindle et al., J Electronic Imaging 10:593-600, 2001.

[4] Y. Ma and M. Wang, Performance of a high-speed impedance camera for flow informatics, Proc. ASME Conference on Engineering Systems Design and Analysis, 19-22 July 2004, Manchester, UK [5] C. A. Garcia-Stewart et al., Proc. 3rd World Congress on Industrial Process Tomography, Banff, Canada, 2003, p. 80-85.

[6] R. M. West et al., Measurement Science and Technology 13:1890-1897 (2002).

[7] H. I. Schlaberg et al, Ultrasonics 38:813-816 (2000).

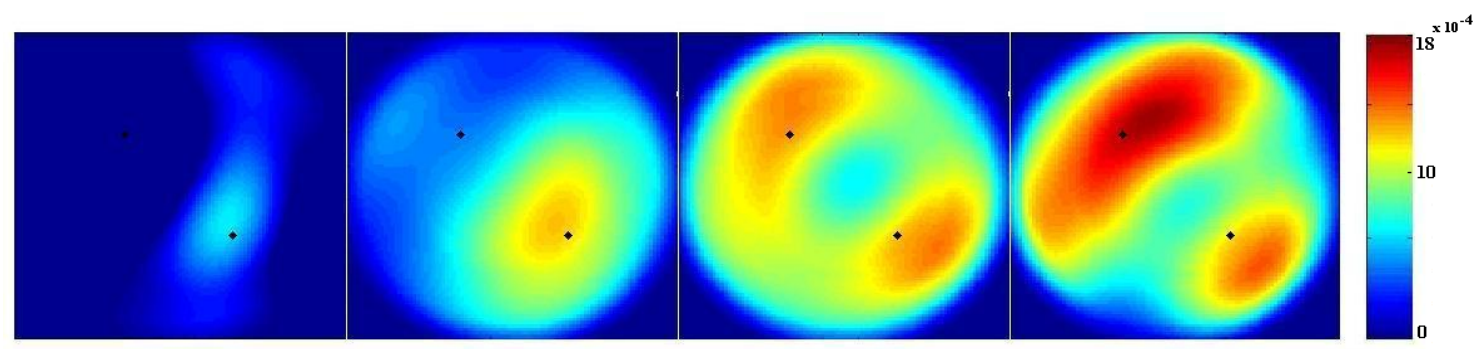

Fig. 1: Sequence of NIR absorption tomograms showing concentration of propane during injection into an engine cylinder (plan view). The locations of both injector ports are shown by a black dot. The images [5] correspond to $11.8 \mathrm{~ms}, 102.9 \mathrm{~ms}, 135.9 \mathrm{~ms}$ and $142.4 \mathrm{~ms}$ after the start of injection.

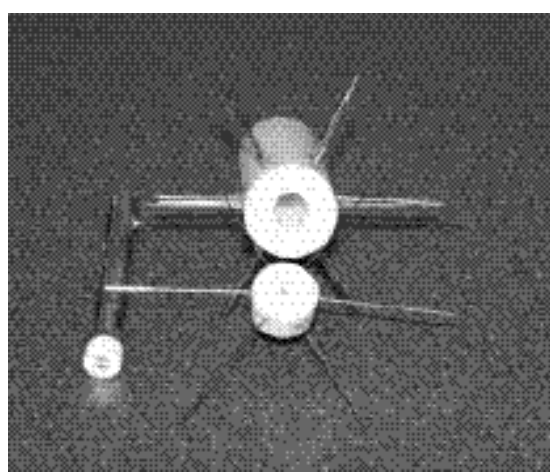

Fig. 2: EIT electrodes in the body and die of a paste extruder [6].

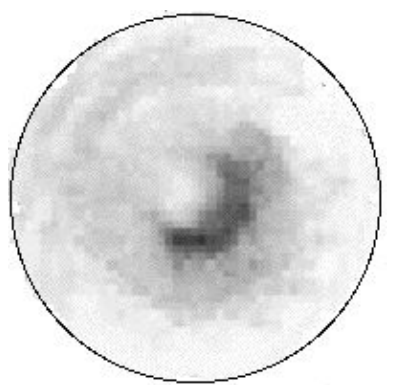

Fig. 4: Ultrasonic attenuation tomogram of the air core created at the center of an industrial $50 \mathrm{~mm}$ diameter hydrocyclone during operation [7]. The air core diameter appears to be about $14 \mathrm{~mm}$. 